\title{
ESTIMATION OF THE MEAN NORMAL MEASURE FROM FLAT SECTIONS
}

\author{
MARKUS KIDERLEN, ${ }^{*}$ University of Aarhus
}

\begin{abstract}
We discuss the determination of the mean normal measure of a stationary random set $Z \subset \mathbb{R}^{d}$ by taking measurements at the intersections of $Z$ with $k$-dimensional planes. We show that mean normal measures of sections with vertical planes determine the mean normal measure of $Z$ if $k \geq 3$ or if $k=2$ and an additional mild assumption holds. The mean normal measures of finitely many flat sections are not sufficient for this purpose. On the other hand, a discrete mean normal measure can be verified (i.e. an a priori guess can be confirmed or discarded) using mean normal measures of intersections with $m$ suitably chosen planes when $m \geq\lfloor d / k\rfloor+1$. This even holds for almost all $m$-tuples of $k$-dimensional planes are viable for verification. A consistent estimator for the mean normal measure of $Z$, based on stereological measurements in vertical sections, is also presented.
\end{abstract}

Keywords: Random set; anisotropy; oriented mean normal measure; rose of normal directions; spherical projection; vertical sections; verification

2000 Mathematics Subject Classification: Primary 60D05

Secondary 53C65; 52A 22

\section{Introduction}

Local first-order properties of a stationary random surface are completely described by its surface area density and its rose of normal directions. The surface area density is a real-valued quantity given as the mean surface area of the random surface per unit volume. The rose of normal directions is a measure on the unit sphere and can be interpreted as the distribution of the unit normal of the surface at a typical point. This distribution has been used to describe (average) anisotropy properties of the random surface, and a number of stereological procedures have been suggested to estimate this measure from flat sections; see [1, Chapter 5], [2], [28, Chapter 9], and the references therein. Actually, all estimation procedures first yield the unoriented mean normal measure, which is the product of the surface area density and the rose of normal directions. An estimator for the rose of normal directions is then simply obtained by normalization. For sufficiently regular stationary random sets $Z$, the rose of normal directions is often defined as the rose of the boundary $\partial Z$ of $Z$; see, e.g. [18]. However, not all kinds of anisotropy can be determined using the unoriented mean normal measure. Consider, for example, the random surface consisting of the union of small circles in the plane, arranged in horizontal rows. This random set clearly exhibits anisotropy, but its rose of normal directions is uniform. This kind of anisotropy, owing to systematic spatial displacements, was formalized and studied in [27]. Even if the displacement is not systematic, the rose of normal directions can be uniform, although, intuitively, the process is not isotropic. Consider, for example,

Received 30 July 2007; revision received 3 December 2007.

* Postal address: Department of Mathematical Sciences, University of Aarhus, Ny Munkegade Build. 1530, DK8000 Aarhus C, Denmark. Email address: kiderlen@imf.au.dk 

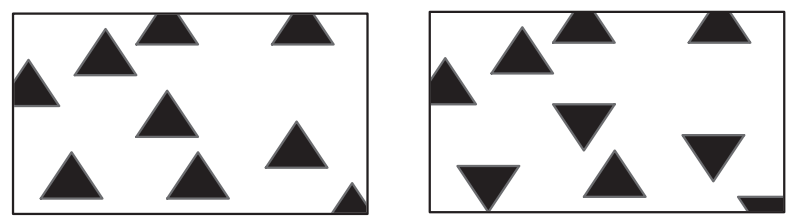

FIGURE 1: Two realizations of planar stationary random sets in a rectangular sampling window with different (oriented) mean normal measures, but coinciding unoriented mean normal measures.

the boundary $\partial Z$ of a stationary Boolean model $Z$ in the plane whose typical particle is a deterministic Reuleaux triangle (an equilateral triangle $T$ whose sides are replaced by circular arcs with endpoints at two vertices of $T$ and centered at the third vertex). In this case the rose of normal directions is uniform, as it does not distinguish between the inner and outer unit normals at a boundary point.

Weil [31] introduced a variant of the unoriented mean normal measure, the mean normal measure $\bar{S}(Z, \cdot)$ of a stationary random set $Z$ in the extended convex ring, taking only the outer unit normals into account. (Schneider [24] called $\bar{S}(Z, \cdot)$ ) the oriented mean normal measure of $Z$.) The mean normal measure was previously considered in [17], [20], and [30] for Boolean models and further treated in [32] and [33]. In the above example, where $Z$ is a Boolean model with a Reuleaux triangle as the typical particle, $\bar{S}(Z, \cdot)$ is not uniform. Figure 1 illustrates that the mean normal measure can also be used to distinguish between certain random sets, even if the unoriented mean normal measures coincide.

The purpose of the present article is to discuss how $\operatorname{far} \bar{S}(Z, \cdot)$ is determined from information in lower-dimensional sections and to suggest an estimator for $\bar{S}(Z, \cdot)$ using a stereological procedure based on vertical sections. Similar uniqueness questions were dealt with in [13], but there, the intersection planes $L$ were considered to be uniform random and connections between certain means of the mean normal measures $\bar{S}^{\prime}(Z \cap L, \cdot)$ of $Z \cap L$ (with respect to $L)$ and $\bar{S}(Z, \cdot)$ were discussed. The prime notation indicates that the mean normal measure is taken relative to $L$; see also Section 2. In contrast to the unoriented mean normal measure, the oriented measure $\bar{S}(Z, \cdot)$ cannot be recovered from one-dimensional sections alone, even if the distributions of the random sets $Z \cap g$ are known for all lines $g$; see [13]. Flat sections of dimension $k$ are sufficient for this purpose if $k$ is at least equal to two. It was shown in [7] that the mean normal measures $\bar{S}^{\prime}(Z \cap L, \cdot)$ determine $\bar{S}(Z, \cdot)$ if $L$ runs through the family of all $k$-dimensional planes. It will be shown in Corollary 3.1 of Section 3 that we can even restrict to planes containing a given direction $u$ (vertical sections) if $k \geq 3$ or if $k=2$ and a mild additional assumption is satisfied. The mean normal measures of finitely many intersections are not sufficient to determine $\bar{S}(Z, \cdot)$. On the other hand, a discrete mean normal measure can be verified (i.e. an a priori guess can be confirmed or discarded) using the mean normal measures of $m$ intersections with $k$-dimensional planes, whenever $m \geq\lfloor d / k\rfloor+1$. This even holds for almost all $m$-tuples of $k$-dimensional planes. This is made precise in Theorem 3.2.

In Section 4 we discuss a procedure to estimate the mean normal measure. In the planar case other approaches have been suggested. Weil [30] gave a method for sets with a polygonal boundary. He also suggested an estimator that uses the additivity of the support function. Rataj [20] constructed an estimator of certain (mean) mixed areas based of area dilations by suitable test sets. From these mixed areas he derived an estimate of the mean normal 
measure. In some practical applications only digitizations of the random set $Z$ are available. Estimators for the mean normal measure from digital images can be found in [14] for the planar case and in [9] and [34] for three-dimensional sets. The only other estimation procedure in higher dimensions was suggested by Schneider in [24]. He used classical stereological information from intersections with pairs of parallel hyperplanes to estimate the mean normal measure of a stationary particle process. This method, however, relies on the inversion of an integral transform, for which no discrete inversion algorithm is known. In Section 4 we present an estimator which is based on the analysis of vertical sections of $Z$. For each vertical section plane $L$, certain intersection points of $\partial(Z \cap L)$ with test lines in $L$ are counted. A twofold application of a known algorithm to invert the cosine transform then yields a discrete nonparametric estimator for $\bar{S}(Z, \cdot)$.

In the next section we give a more formal definition of the mean normal measure and recall relevant integral formulae connecting it to flat sections of $Z$.

\section{The mean normal measure}

We introduce some notation. The Euclidean norm and the inner product in $\mathbb{R}^{d}$ are denoted by $\|\cdot\|$ and $\langle\cdot, \cdot\rangle$, respectively, the unit ball is denoted by $B^{d}$, and the unit sphere is denoted by $S^{d-1}=\partial B^{d}$. Let $u^{\perp}$ be the linear hyperplane with unit normal $u \in S^{d-1}$. We will often use the great sphere $u^{\circ}:=u^{\perp} \cap S^{d-1}$ orthogonal to $u$ and the relative open half spheres generated by it. Set

$$
u^{\oplus}:=\left\{v \in S^{d-1}:\langle u, v\rangle>0\right\}
$$

and $u^{\ominus}:=(-u)^{\oplus}$. Let $\mathcal{L}_{k}^{d}$ be the Grassmannian of all $k$-dimensional linear subspaces of $\mathbb{R}^{d}$, and let $v_{k}$ be the rotation invariant probability measure on this space. We write

$$
\mathcal{L}_{k}^{d}(u):=\left\{L \in \mathcal{L}_{k}^{d}: u \in L\right\}
$$

for the sheaf of all $k$-dimensional subspaces containing the direction $u$. For a measure $\mu$ defined on a $\sigma$-algebra $\mathcal{B}$ and $B \in \mathcal{B}$, we will write $\mu\llcorner B:=\mu(\cdot \cap B)$. Thus, $\mu\llcorner B$ coincides with $\mu$ on all Borel subsets of $B$ and vanishes identically outside $B$. We will always denote by $\mathscr{H}^{m}$ the Hausdorff measure of dimension $m$ in $\mathbb{R}^{d}$. A measure $\mu$ on $S^{d-1}$ is even if it coincides with its even part given by

$$
A \mapsto \frac{1}{2}(\mu(A)+\mu(-A))
$$

for Borel sets $A \subset S^{d-1}$.

Let $\mathcal{K}$ denote the set of convex bodies (compact convex sets) in $\mathbb{R}^{d}$. The support measures (generalized curvature measures) $\Theta_{j}(K, \cdot), j=0, \ldots, d-1$, of $K \in \mathcal{K}$ can be defined as coefficients of a Steiner-type formula for local parallel volumes; see [23, Chapter 4]. As we will use only the support measure of order $j=d-1$, we give a more direct description, which applies to arbitrary closed sets [10], but will here be used only for sets in the extended convex ring. The convex ring $\mathcal{R}$ is the family of all finite unions of convex bodies, and the extended convex ring

$$
s:=\left\{A \subset \mathbb{R}^{d}: A \cap K \in \mathcal{R} \text { for all } K \in \mathcal{K}\right\}
$$

is the family of sets that can locally be written as finite unions of convex bodies. For $K \in \delta$, let $\partial^{+} K$ be the positive boundary consisting of all $x \in \partial K$ that have an outer normal $u \in S^{d-1}$, meaning that there exists a positive $\varepsilon$ such that $x$ is the (unique) metric projection of $x+\varepsilon u$ on $K$. Equivalently, the intersection of the ball $(x+\varepsilon u)+\varepsilon B^{d}$ and $K$ is the singleton $\{x\}$. The set 
$N(K, x)$ of all the outer normals of $K$ at $x \in \partial^{+} K$ contains either one, two antipodal, or infinitely many points. Set

$$
\partial^{i} K:=\left\{x \in \partial^{+} K: \operatorname{card} N(K, x)=i\right\}, \quad i=1,2 .
$$

The unique normal in $x \in \partial^{1} K$ at $K$ is denoted by $u(K, x)$. For $x \in \partial^{2} K$, we select $u(K, x)$ as one of the two possible normals in a measurable manner. As $K \in \&$, we have

$$
\mathscr{H}^{d-1}(\partial K)=\mathscr{H}^{d-1}\left(\partial^{+} K\right)=\mathscr{H}^{d-1}\left(\partial^{1} K \cup \partial^{2} K\right) .
$$

If $K \in \&$ is topologically regular (i.e. it is the closure of its interior) then $\partial^{2} K=\varnothing$. The support measure (of order $d-1$ ) of $K$ is the measure on $\mathbb{R}^{d} \times S^{d-1}$ given by

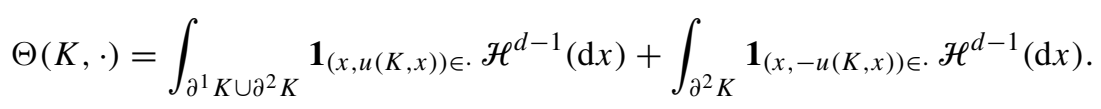

The projection $S(K, \cdot)=\Theta\left(K, \mathbb{R}^{d} \times \cdot\right)$ is the surface area measure (of order $d-1$ ) of $K$. If $K$ is contained in a subspace $L \in \mathcal{L}_{k}^{d}$ with $k \leq d-2$, the curvature measure $\Theta(K, \cdot)$ is trivial. Then we often consider the curvature measure of $K$ as a subset of $L$ and denote it by $\Theta^{\prime}(K, \cdot)$. The subspace $L$ will be clear from the context. Note that $\Theta^{\prime}(K, \cdot)$ is a measure on $L \times S^{k-1}(L)$, where $S^{k-1}(L)=S^{d-1} \cap L$ is the unit sphere in $L$. We denote the orthogonal projection of $x \in \mathbb{R}^{d}$ on $L$ by $x \mid L$. Let

$$
\operatorname{pr}_{L}(u):=\frac{(u \mid L)}{\|u \mid L\|}
$$

be the spherical projection of $u \in S^{d-1}$ on $S^{k-1}(L)$. As $\operatorname{pr}_{L}(u)$ is undefined when $u$ is in the orthogonal complement $L^{\perp}$ of $L$, we set $\operatorname{pr}_{L}(u):=v_{0}$ for some fixed vector $v_{0} \in S^{k-1}(L)$ in this case. Rataj [21] showed a translative Crofton-type formula for sets of positive reach, which, by additivity, also holds for sets $K \in \mathcal{R}$. For $L \in \mathcal{L}_{k}^{d}, k=1, \ldots, d-1$, and any measurable function $f: L^{\perp} \times L \times S^{k-1}(L) \rightarrow \mathbb{R}_{+}$, we have

$$
\begin{gathered}
\int_{L^{\perp}} \int_{L \times S^{k-1}(L)} f(-z, x, u) \Theta^{\prime}((K+z) \cap L, \mathrm{~d}(x, u)) \mathscr{H}^{d-k}(\mathrm{~d} z) \\
=\int_{\mathbb{R}^{d} \times S^{d-1}} f\left(x\left|L^{\perp}, x\right| L, \operatorname{pr}_{L}(u)\right)\|u \mid L\| \Theta(K, \mathrm{~d}(x, u)) .
\end{gathered}
$$

This relation can also be derived from [11, Satz 2.9 and Lemma 2.5] using support measures relative to a gauge body which is not necessarily the Euclidean ball; see also [15]. Specializing (2.1) to surface area measures, we obtain

$$
\int_{L^{\perp}} S^{\prime}((K+z) \cap L, \cdot) \mathscr{H}^{d-k}(\mathrm{~d} z)=\pi_{L} S(K, \cdot),
$$

where $\pi_{L}$ is a linear and weakly continuous operator from the space $\mathcal{M}$ of finite signed Borel measures on $S^{d-1}$ to the space $\mathcal{M}(L)$ of finite signed Borel measures on $S^{k-1}(L)$. For $\mu \in \mathcal{M}$, the measure $\pi_{L} \mu$ is defined as the image measure of

$$
\int_{(\cdot)}\|u \mid L\| \mu(\mathrm{d} u)
$$


under the spherical projection $\operatorname{pr}_{L}$. In particular, if $L=g=\operatorname{lin} v$ is a line with direction $v \in S^{d-1}$, the measure $\pi_{g} \mu$ is concentrated on $\{-v, v\}$ and has total mass

$$
\left(\pi_{g} \mu\right)(\{-v, v\})=C \mu(v),
$$

where

$$
C \mu=\int_{S^{d-1}}|\langle u, \cdot\rangle| \mu(\mathrm{d} u)
$$

is the cosine transform of $\mu$. For later use, we note that

$$
\pi_{M}^{L} \pi_{L}=\pi_{M}
$$

for all nontrivial subspaces $M \subset L$, where $\pi_{M}^{L}$ is the projection operator on the unit sphere in $M$, relative to $L$. The operator $\pi_{L}$ is strongly related to the cosine transform and, hence, to projection bodies (see [3] and [29]). It is a special case of the more general spherical projection operators $\pi_{L, m}$, where the integrand in (2.3) is taken to the power $m$ (see [8] for their properties and use in geometric tomography, and for a proof of (2.5) in terms of $\pi_{L, m}$ ). A generalization of (2.2) for mixed surface area measures was shown in [7].

For a definition and basic properties of random closed sets, we refer the reader to [26, Chapters 1 and 2]. The mean normal measure $\bar{S}(Z, \cdot)$ can be defined for stationary random closed sets in $\mathbb{R}^{d}$ without any further assumption; cf. [10, Section 7]. However, owing to this generality, even the intuitive property that its mean total mass for a topologically regular random set equals the surface area density does not hold. In the following we will consider only stationary random closed sets $Z$ in the extended convex ring $\&$. Many of the results also hold for $U_{\mathrm{PR}}$ sets, that is sets that can be written as locally finite unions of sets of positive reach, which are such that any finite intersection of them has again positive reach. However, as we make extensive use of translative Crofton formulae for surface area measures, this would require additional assumptions on the relative positions of $Z$ and the considered intersection plane; see [21]. Throughout this article, we assume that $Z$ is a stationary set in $\delta$ and has finite mean local surface area. We have

$$
\mathrm{E} \mathscr{H}^{d-1}(\partial Z \cap K)<\infty
$$

for one (and hence all) convex body $K$ with interior points. In the literature the stronger integrability condition

$$
\mathrm{E} 2^{N(Z \cap K)}<\infty
$$

is sometimes assumed, where $N(M)$ is the minimal number of convex bodies needed to write the nonempty set $M \in \mathcal{R}$ as their union, and $N(\varnothing)=0$. We will not require (2.7). For $K \in \mathcal{K}$ with the origin in its interior, Weil [31] defined the mean normal measure of $Z$ by

$$
\bar{S}(Z, \cdot):=\lim _{r \rightarrow \infty} \frac{1}{\mathscr{H}^{d}(r K)} \mathrm{E} S(Z \cap r K, \cdot),
$$

which is independent of $K$. Moreover,

$$
\bar{S}(Z, \cdot)=\mathrm{E}\left(S\left(Z \cap C^{d}, \cdot\right)-S\left(Z \cap C_{1}^{d}, \cdot\right)\right),
$$

where $C^{d}$ is the unit cube in $\mathbb{R}^{d}$ and

$$
C_{1}^{d}=\left\{x=\left(x_{1}, \ldots, x_{d}\right) \in C^{d}: \max _{i=1, \ldots, d} x_{i}=1\right\}
$$


is its 'upper-right boundary'. A third representation of the mean normal measure is

$$
\bar{S}(Z, \cdot)=\frac{1}{\mathscr{H}^{d}(K)} \mathrm{E} \Theta(Z ;(\text { int } K) \times \cdot),
$$

where $K$ is an arbitrary convex body with interior points. Equation (2.8) follows from a special case of a translative integral formula for support measures in [22]. In [31] condition (2.7) was assumed, but this condition can be replaced by the weaker condition (2.6), as, for any Borel set $A \subset S^{d-1}$

$$
0 \leq S(Z \cap K, A) \leq 2 \mathscr{H}^{d-1}(\partial Z \cap K),
$$

where the right-hand side is monotone in $K$ with respect to set inclusion.

A combination of (2.8) and (2.1) implies that

$$
\bar{S}^{\prime}(Z \cap L, \cdot)=\pi_{L} \bar{S}(Z, \cdot) .
$$

This was shown by Weil [31] (see also [32]) and is the starting point for our uniqueness results.

\section{Determination of the mean normal measure}

Fix $k \in\{2, \ldots, d-1\}$. It was shown in [7] that a finite signed measure $\mu$ on the unit sphere is uniquely determined by the family of all its projections $\pi_{L} \mu, L \in \mathcal{L}_{k}^{d}$. In view of (2.9) and the linearity of $\pi_{L}$, this shows that the mean normal measure $\bar{S}(Z, \cdot)$ is uniquely determined by all mean normal measures of sections $\bar{S}^{\prime}(Z \cap L, \cdot), L \in \mathcal{L}_{k}^{d}$. Our first result states that the latter family actually contains considerable redundant information, as we can restrict to vertical planes, i.e. planes that contain a line with a given direction $u \in S^{d-1}$, at least when $k \geq 3$. Its proof is based on injectivity properties of the cosine transform and avoids the use of mixed volumes and spherical harmonics which are the basis of the uniqueness result in [7]. We will write $\boldsymbol{o}$ for the zero measure.

Theorem 3.1. Let a finite signed measure $\mu$ on the unit sphere, $k \in\{2, \ldots, d-1\}$, and $u \in S^{d-1}$ be given. If $k \geq 3$ then

$$
\pi_{L} \mu=\boldsymbol{o} \text { for all } L \in \mathcal{L}_{k}^{d}(u),
$$

implies that $\mu=\boldsymbol{o}$. For $k=2$, the same implication holds true when $\mu\left\llcorner u^{\circ}\right.$ is even.

Proof. We first fix $v \in S^{d-1}$ and $m \geq 2$, and show an intermediate claim. If

$$
\pi_{M} \mu=\boldsymbol{o} \text { for all } M \in \mathcal{L}_{m}^{d}(v),
$$

then $\mu\left\llcorner v^{\oplus}=\boldsymbol{o}\right.$. In fact, as a unit vector is in $v^{\oplus}$ if and only if its spherical projection on $M$ is in $v^{\oplus} \cap M,(3.2)$ implies that

$$
\pi_{M}\left(\mu\left\llcorner v^{\oplus}\right)=\left(\pi_{M} \mu\right)\left\llcorner\left(v^{\oplus} \cap M\right)=\boldsymbol{o} .\right.\right.
$$

Hence, if $g$ is an arbitrary line in $M$ and we abbreviate $\mu^{\oplus}:=\mu\left\llcorner v^{\oplus},(2.5)\right.$ gives

$$
\pi_{g}\left(\mu^{\oplus}\right)=\pi_{g}^{M}\left(\pi_{M} \mu^{\oplus}\right)=\boldsymbol{o} .
$$

As $g$ was arbitrary in $M$, which in turn was arbitrary in $\mathcal{L}_{m}^{d}(v),(2.4)$ implies that the cosine transform $C \mu^{\oplus}$ of $\mu^{\oplus}$ vanishes on $S^{d-1}$. The cosine transform determines the even part of a 
measure; see [23, Theorem 3.5.3 and Note 3 on p. 192]. As $\mu^{\oplus}$ is concentrated on a relative open half sphere, we find that $\mu^{\oplus}=\boldsymbol{o}$ and the intermediate claim is shown.

Now let the assumptions of the theorem be satisfied with $k \geq 2$. The intermediate claim with $m=k$ and $v= \pm u$ implies that

$$
\mu\left\llcorner\left(u^{\oplus} \cup u^{\ominus}\right)=\boldsymbol{o},\right.
$$

and it is enough to show that the restriction $\mu^{\circ}$ of $\mu$ to the Borel sets in $u^{\circ}$ vanishes. For $L \in \mathcal{L}_{k}^{d}(u)$ and $M=L \cap u^{\perp}$, we have $\operatorname{pr}_{L}=\operatorname{pr}_{M}$ on $u^{\circ}$, so (3.3) and (3.1) imply that

$$
\pi_{M}^{u^{\perp}}\left(\mu^{\circ}\right)=\pi_{L} \mu=\boldsymbol{o} .
$$

Consider the case in which $k \geq 3$. As $L$ can be chosen arbitrarily, (3.4) is true for all subspaces $M$ of $u^{\perp}$ of dimension $k-1 \geq 2$. Thus, the intermediate claim can be applied in $u^{\perp}$ with $m=k-1, \mu$ replaced by $\mu^{\circ}$, and arbitrary $v \in u^{\circ}$. We obtain $\mu^{\circ}\left\llcorner\left(v^{\oplus} \cap u^{\circ}\right)=\boldsymbol{o}\right.$ for all $v \in u^{\circ}$, which clearly gives the desired result $\mu=\boldsymbol{o}$.

For $k=2, M$ in (3.4) is a line and (2.4) applied in $u^{\perp}$ together with the injectivity of the cosine transform on even measures implies that the even part of $\mu^{\circ}$ must be 0 . As we assumed that $\mu^{\circ}$ was even, this gives $\mu^{\circ}=\boldsymbol{o}$, as required.

Owing to (2.9), we may rephrase this result in terms of the mean normal measure.

Corollary 3.1. Let $Z$ be a stationary random set in the extended convex ring in $\mathbb{R}^{d}$ such that (2.6) holds. For fixed $u \in S^{d-1}$, the mean normal measure $\bar{S}(Z, \cdot)$ is uniquely determined by the mean normal measures $\bar{S}^{\prime}(Z \cap L, \cdot)$, where $L$ runs through the sheaf of planes $\mathcal{L}_{k}^{d}(u)$ if $k \geq 3$.

The same holds true if $k=2$ and $\bar{S}(Z, \cdot)\left\llcorner u^{\circ}\right.$ is even.

Some comments on the case in which $k=2$ are relevant to mention here, as ordinary planes in $\mathbb{R}^{3}$ are the most important case for applications. Firstly, the additional condition for $k=2$ is obviously fulfilled when $\bar{S}(Z, \cdot)\left\llcorner u^{\circ}=\boldsymbol{o}\right.$, and this is true in particular if $\bar{S}(Z, \cdot)$ is absolutely continuous with respect to the spherical Lebesgue measure. If this cannot be assumed, we can randomize the choice of $u$. Whenever $u$ is chosen as a random isotropic direction on $S^{d-1}$ (independent of $Z$ ), then $\bar{S}(Z, \cdot)\left\llcorner u^{\perp}=\boldsymbol{o}\right.$ holds almost surely. Thus, the uniqueness result also holds almost surely. If extra assumptions are to be avoided, we can consider one additional section with a hyperplane that does not contain the axis of the sheaf. With essentially the same arguments that led to Theorem 3.1, it can be shown that $\bar{S}(Z, \cdot)$ is uniquely determined by the collection of measures

$$
\bar{S}^{\prime}(Z \cap L, \cdot), \quad L \in \mathcal{L}_{2}^{d}(u) \cup\left\{L_{0}\right\},
$$

where $L_{0} \in \mathcal{L}_{d-1}^{d}$ satisfies $u \notin L_{0}$.

The question arises whether finitely many sections are enough to determine the mean normal measure. This is not the case, as we will show below. On the other hand, discrete mean normal measures can be verified using finitely many (actually very few) suitably chosen flat sections. To prove these results, we will describe the action of $\pi_{L}$ on discrete measures in a more geometric way, apply tools from geometric tomography, and transfer the obtained statements to mean normal measures of random sets.

We call a measure discrete if its support is at most countable. Let $\mathcal{M}_{d}$ denote the space of all discrete finite signed measures on $S^{d-1}$ and $\mathcal{M}_{d}^{+}$denote the subcone of positive measures in $\mathcal{M}_{d}$. We write $\delta_{u}$ for the Dirac point measure supported by $u \in S^{d-1}$. Any measure $\mu \in \mathcal{M}_{d}^{+}$is of 
the form $\mu=\sum_{i=1}^{N} \alpha_{i} \delta_{u_{i}}$ with positive masses $\alpha_{i}$, pairwise different support points $u_{1}, u_{2}, \ldots$ in $S^{d-1}, N \in \mathbb{N}$ or $N=\infty$, and $\sum_{i=1}^{N} \alpha_{i}<\infty$. The idea that spherical projections of discrete measures are closely related to orthogonal projections of associated point sets in $\mathbb{R}^{d}$ was pointed out to us by W. Weil and is expressed formally by (3.6), below. For $\mu$ as above, we set

$$
\mathcal{P}(\mu):=\bigcup_{i=1}^{N}\left\{\alpha_{i} u_{i}\right\} .
$$

This gives rise to a bijection from $\mathcal{M}_{d}^{+}$onto the family $\mathcal{F}$ of at most countable sets $F \subset \mathbb{R}^{d} \backslash\{0\}$ with the properties that

(a) any ray emanating from 0 hits $F$ in at most one point;

(b) $\sum_{x \in F}\|x\|<\infty$.

Let $L \in \mathcal{L}_{k}^{d}, 1 \leq k \leq d-1$. The definition of $\pi_{L}$ implies that

$$
\pi_{L} \mu=\sum_{x \in L \backslash\{0\}}\|x\| X_{\mathcal{P}(\mu)}(x) \delta_{x /\|x\|},
$$

where

$$
X_{A}(x)=\operatorname{card}\left(A \cap\left(x+L^{\perp}\right)\right), \quad x \in L,
$$

is the (discrete) $X$-ray function of $A \subset \mathbb{R}^{d}$ in direction $L^{\perp}$. Thus, $\pi_{L} \mu$ is uniquely determined by the X-ray function of $\mathcal{P}(\mu)$. The converse is true for $L$ in

$$
\begin{gathered}
\mathcal{N}_{k}^{d}(\mu):=\left\{M \in \mathcal{L}_{k}^{d}: \operatorname{pr}_{M}\left(u_{1}\right), \ldots, \operatorname{pr}_{M}\left(u_{N}\right)\right. \text { are pairwise different and } \\
\left.M^{\perp} \cap\left\{u_{1}, \ldots, u_{N}\right\}=\varnothing\right\} .
\end{gathered}
$$

For $L \in \mathcal{N}_{k}^{d}(\mu)$, we have $X_{\mathcal{P}(\mu)}=\mathbf{1}_{\mathcal{P}(\mu) \mid L}$ on $L$ and

$$
\mathcal{P}\left(\pi_{L} \mu\right)=\mathscr{P}(\mu) \mid L .
$$

Note that

$$
v_{k}\left(\mathcal{N}_{k}^{d}(\mu)\right)=1 \quad \text { if } 2 \leq k \leq d-1
$$

for all $\mu \in \mathcal{M}_{d}^{+}$, whereas $\mathcal{N}_{1}^{d}(\mu)=\varnothing$ if the support of $\mu$ consists of at least three points. Surface area measures of convex bodies with interior points have this property for $d \geq 2$, which is the reason that (3.6) is of interest only for $k \geq 2$.

We now ask whether a discrete measure $\mu$ is determined by spherical projections on subspaces in a given finite set $\mathcal{L} \subset \mathcal{L}_{k}^{d}$. Owing to (3.6) and the injectivity of $\mathcal{P}$, this question can be rephrased to asking whether a discrete set in $\mathbb{R}^{d}$ is determined by all X-ray functions on planes in $\mathcal{L}$. Discrete tomography yields an answer to this question.

Proposition 3.1. Let $1 \leq k \leq d-1$, and let $\mathcal{L} \subset \mathcal{L}_{k}^{d}$ be finite. Then there exist two different finite measures $\mu$ and $\mu^{\prime}$, indistinguishable by their spherical projections on the members of $\mathcal{L}$ :

$$
\pi_{L} \mu=\pi_{L} \mu^{\prime}
$$

for all $L \in \mathcal{L}$. These measures can be chosen even and with finite supports. 
Proof. Owing to (2.5), it is enough to consider hyperplanes $L$. Let $\mathcal{L}=\left\{L_{1}, \ldots, L_{m}\right\} \subset$ $\mathcal{L}_{d-1}^{d}$. According to [4, Lemma 2.3.2], there exists an $\left\{L_{1}^{\perp}, \ldots, L_{m}^{\perp}\right\}$-switching component. This is a union $A \cup A^{\prime}$ of finite, disjoint, and nonempty sets $A$ and $A^{\prime}$ with

$$
\operatorname{card}\left(A \cap\left(x+L_{i}^{\perp}\right)\right)=\operatorname{card}\left(A^{\prime} \cap\left(x+L_{i}^{\perp}\right)\right) \text { for all } x \in \mathbb{R}^{d}, i=1, \ldots, m .
$$

Hence, $A$ and $A^{\prime}$ have the same discrete X-ray functions in the directions $L_{1}^{\perp}, \ldots, L_{m}^{\perp}$, although they are disjoint. Equation (3.7) is invariant with respect to arbitrary translations of $A \cup A^{\prime}$. It is also unchanged when $A \cup A^{\prime}$ is reflected at the origin. We can therefore assume that

$$
A \cap \bigcup_{i=1}^{m} L_{i}^{\perp}=A^{\prime} \cap \bigcup_{i=1}^{m} L_{i}^{\perp}=\varnothing,
$$

every ray starting at 0 hits $A \cup A^{\prime}$ in at most one point, and that $A$ and $A^{\prime}$ are both origin symmetric. These conditions imply that there exist finite even measures $\mu$ and $\mu^{\prime}$ with $\mathcal{P}(\mu)=A$ and $\mathcal{P}\left(\mu^{\prime}\right)=A^{\prime}$ with disjoint finite supports. Owing to (3.5), the spherical projections of these measures can be expressed using the X-ray functions of $A$ and $A^{\prime}$, which coincide by (3.7). We thus obtain $\pi_{L_{i}} \mu=\pi_{L_{i}} \mu^{\prime}$ for all $i=1, \ldots, m$.

Gardner [4, p. 64] introduced the notion of verification of sets by X-rays. We transfer this notion to the present context for measures in a subcone $\mathcal{M}^{\prime}$ of $\mathcal{M}$. A measure $\mu \in \mathcal{M}^{\prime}$ can be verified by spherical projections on $m$ planes in $\mathcal{L}_{k}^{d}$ if $L_{1}, \ldots, L_{m} \in \mathcal{L}_{k}^{d}$ can be chosen (depending on $\mu$ ) such that if $\mu^{\prime} \in \mathcal{M}^{\prime}$ and $\pi_{L_{i}} \mu=\pi_{L_{i}} \mu^{\prime}$ for $1 \leq i \leq m$ then $\mu=\mu^{\prime}$. We say that a measure $\mu \in \mathcal{M}^{\prime}$ can almost surely be verified by $m$ spherical projections on planes in $\mathcal{L}_{k}^{d}$ if, for all $\left(L_{1}, \ldots, L_{m}\right) \in \mathcal{L}_{k}^{d} \times \cdots \times \mathcal{L}_{k}^{d}$ with the possible exception of a zero measure set of $v_{k}^{\otimes m}$, again, $\mu^{\prime} \in \mathcal{M}^{\prime}$ and $\pi_{L_{i}} \mu=\pi_{L_{i}} \mu^{\prime}$ for $1 \leq i \leq m$ implies that $\mu=\mu^{\prime}$. Note that the zero measure set may depend on $\mu$. This subtle dependence on $\mu$ is the reason for the use of the term verification instead of determination; cf. [4, p. 64]. Clearly, almost sure verification is stronger than ordinary verification.

Proposition 3.2. Let $2 \leq k \leq d-1$, and let $m=\lfloor d / k\rfloor+1$. Then any $\mu \in \mathcal{M}_{d}^{+}$can almost surely be verified by $m$ spherical projections on planes in $\mathcal{L}_{k}^{d}$. The value of $m$ is best possible here.

Proof. Let $\mu \in \mathcal{M}_{d}^{+}$, let $F:=\mathcal{P}(\mu)$, and let $m=\lfloor d / k\rfloor+1$. We modify the proof of [5, Theorem 7.4] appropriately. We first choose $m-1$ subspaces $L_{1}, \ldots, L_{m-1} \in \mathcal{N}_{k}^{d}(\mu)$ such that $L_{1}^{\perp}, \ldots, L_{m-1}^{\perp}$ are in general position. Note that $v_{k}^{\otimes(m-1)}$-almost all $\left(L_{1}, \ldots, L_{m-1}\right)$ satisfy these conditions. The subspace $T:=\bigcap_{i=1}^{m-1} L_{i}^{\perp}$ has dimension

$$
\operatorname{dim} T=(m-1)(d-k)-(m-2) d=d-\left\lfloor\frac{d}{k}\right\rfloor k<k .
$$

The set

$$
G:=\bigcap_{i=1}^{m-1}\left\{x+L_{i}^{\perp}: x \in F \mid L_{i}\right\}
$$

is a finite union of translates of $T, G=\left\{y_{1}+T, \ldots, y_{s}+T\right\}$, say. Let $L_{i j}$ be the maximal linear subspace parallel to the affine hull of $\left(y_{i}+T\right) \cup\left(y_{j}+T\right), 1 \leq i<j \leq s$. As $\operatorname{dim} T<k$, we have $\operatorname{dim} L_{i j} \leq k$ and almost all $L_{m} \in \mathcal{L}_{k}^{d}$ have the property that $L_{m}^{\perp}$ intersects $L_{i j}$ in one point for all $1 \leq i<j \leq s$. Hence, all $(d-k)$-dimensional planes $x+L_{m}^{\perp}, x \in \mathbb{R}^{d}$, intersect 
at most one of the translates of $T$ in $G$, and each of these intersections is a single point. Fix one of these subspaces $L_{m} \in \mathcal{N}_{k}^{d}(\mu)$. It is easy to see that

$$
F=\bigcap_{i=1}^{m}\left\{x+L_{i}^{\perp}: x \in F \mid L_{i}\right\},
$$

where the right-hand side is determined by the spherical projections of $\mu$ owing to (3.6). But $F=\mathcal{P}(\mu)$ determines $\mu$. Note that $\left(L_{1}, \ldots, L_{m}\right)$ was chosen arbitrarily in $\mathcal{L}_{k}^{d} \times \cdots \times \mathcal{L}_{k}^{d}$ excluding only a zero measure set (which may depend on $\mu$ ).

It remains to show that the number $m$ is best possible. In the proof of [5, Theorem 7.4] the existence of a finite set $F \subset \mathbb{R}^{d}$ (being actually the vertex set of a zonotope with interior points) is shown such that, for any set $\mathcal{L} \subset \mathcal{L}_{k}^{d}$ of $\lfloor d / k\rfloor$ subspaces, there exists a different finite set $F^{\prime} \subset \mathbb{R}^{d}$ with the same X-rays as $F$ in directions $L^{\perp}$ for all $L \in \mathcal{L}$. The two measures $\mu$ and $\mu^{\prime}$ with $\mathcal{P}(\mu)=F$ and $\mathcal{P}\left(\mu^{\prime}\right)=F^{\prime}$ show that $m$ is optimal.

In view of (2.9) the above results can be reformulated in terms of random sets. We call a random set $Z$ polyhedral if it is almost surely a locally finite union of convex polytopes. Typical examples of polyhedral sets are crystalline media. Restricting further, we call a random set $Z$ simple polyhedral if there exists an at most countable set of unit vectors containing almost surely all facet unit normals of $Z$. A stationary random set $Z$ is simple polyhedral if and only if $\bar{S}(Z, \cdot)$ is discrete. The random sets in part (a) of Theorem 3.2, below, can actually be chosen to be Boolean models (with deterministic grains); see [26, Section 4.4] for a definition.

Theorem 3.2. (a) For any $1 \leq k \leq d-1$ and any finite $\mathcal{L} \subset \mathcal{L}_{k}^{d}$, there exist two stationary simple polyhedral random sets $Z$ and $Z^{\prime}$ with different mean normal measures, but such that

$$
\bar{S}^{\prime}(Z \cap L, \cdot)=\bar{S}^{\prime}\left(Z^{\prime} \cap L, \cdot\right)
$$

holds for all $L \in \mathcal{L}$.

(b) Let $2 \leq k \leq d-1$, let $m=\lfloor d / k\rfloor+1$, and let $Z$ be a stationary simple polyhedral random set obeying (2.6). Then, for $v_{k}^{\otimes m}$-almost all $\left(L_{1}, \ldots, L_{m}\right) \in\left(\mathcal{L}_{k}^{d}\right)^{m}$ and all stationary simple polyhedral random sets $Z^{\prime}$,

$$
\bar{S}^{\prime}\left(Z \cap L_{i}, \cdot\right)=\bar{S}^{\prime}\left(Z^{\prime} \cap L_{i}, \cdot\right), \quad i=1, \ldots, m,
$$

implies that $\bar{S}(Z, \cdot)=\bar{S}\left(Z^{\prime}, \cdot\right)$.

Proof. As part (b) is a direct consequence of Proposition 3.2 and (2.9), it remains to show part (a). According to Proposition 3.1, there exist two different finite even measures $\mu$ and $\mu^{\prime}$ with finite supports such that $\pi_{L} \mu=\pi_{L} \mu^{\prime}$ holds for all $L \in \mathcal{L}$. We may assume that the supports of $\mu$ and $\mu^{\prime}$ are not concentrated on great circles of $S^{d-1}$ (otherwise, add a suitable even measure in $\mathcal{M}_{d}^{+}$to both). Owing to Minkowski's existence theorem (see, e.g. [23, Section 7.1]), there exist two convex polytopes $K$ and $K^{\prime}$ (origin symmetric and with interior points) with $S(K, \cdot)=\mu$ and $S\left(K^{\prime}, \cdot\right)=\mu^{\prime}$. Now let $Z$ and $Z^{\prime}$ be stationary Boolean models with deterministic typical grains $K$ and $K^{\prime}$, respectively. Their positive intensities $\gamma$ and $\gamma^{\prime}$ depend on the volumes $V(K)$ and $V\left(K^{\prime}\right)$ of $K$ and $K^{\prime}$ and are chosen such that

$$
\gamma \mathrm{e}^{-\gamma V(K)}=\gamma^{\prime} \mathrm{e}^{-\gamma^{\prime} V\left(K^{\prime}\right)}=: \alpha .
$$


Using [33, Section 3.2], we see that

$$
\bar{S}(Z, \cdot)=\gamma \mathrm{e}^{-\gamma V(K)} S(K, \cdot)=\alpha \mu
$$

and $\bar{S}\left(Z^{\prime}, \cdot\right)=\alpha \mu^{\prime}$. Hence, $\bar{S}(Z, \cdot) \neq \bar{S}\left(Z^{\prime}, \cdot\right)$, but

$$
\bar{S}^{\prime}(Z \cap L, \cdot)=\alpha \pi_{L} \mu=\alpha \pi_{L} \mu^{\prime}=\bar{S}^{\prime}\left(Z^{\prime} \cap L, \cdot\right)
$$

holds for all $L \in \mathcal{L}$ according to (2.9).

\section{Estimation of the mean normal measure from vertical sections}

From a stereological point of view, an estimation procedure for $\bar{S}(Z, \cdot)$ should only require simple data acquisition such as measurements of lengths or counting. Schneider [24] suggested using surface area measurements in intersections with hyperplanes. He considered the mean normal measure $\bar{S}(X, \cdot)$ of a stationary particle process $X$ of convex particles, and showed that the family of densities

$$
\overline{\mathscr{H}}^{d-2}\left(\partial^{u} X \cap u^{\perp}\right), \quad u \in S^{d-1},
$$

determined $\bar{S}(X, \cdot)$ uniquely. Here $\overline{\mathscr{H}}^{d-2}$ denotes the density of the $(d-2)$-dimensional Hausdorff measure, and $\partial^{u} X$ is the surface process of all boundary points of $X$-particles having an outer unit normal in $S^{d-1} \cap u^{\oplus}$ (Schneider actually worked with normals in the closed half sphere $S^{d-1} \backslash u^{\ominus}$, which led to the same densities for almost all $u$ ). He also suggested estimating $\overline{\mathscr{H}}^{d-2}\left(\partial^{u} X \cap u^{\perp}\right)$ using a pair of parallel hyperplanes at small distance to avoid an explicit determination of the outer normal in $\mathbb{R}^{d}$. His uniqueness proof was based on injectivity properties of the hemispherical sine transform. As there is no discrete algorithm available which allows us to invert this integral transform, the suggested method is of limited value for applications, at least for the time being.

Here we suggest a method that is based on vertical sections in the spirit of the proof of Theorem 3.1. It allows us to replace the inversion of the hemispherical sine transform by the inversion of the cosine transform, for which nonparametric algorithms are known. The common direction $u$ of the sheaf of vertical intersection planes will be considered fixed and called the reference direction in the following. For $L \in \mathcal{L}_{k}^{d}(u)$, let $\partial_{L}^{u}(Z \cap L)$ denote the set of all boundary points of $Z \cap L$ (relative to $L$ ) that have an outer unit normal in $S^{k-1}(L) \cap u^{\oplus}$. For a test line $g=\operatorname{lin} v \subset L, v \in S^{k-1}(L)$, and a measurable set $W \subset g$ with $0<\mathscr{H}^{1}(W)<\infty$, set

$$
\gamma^{+}(v):=\frac{1}{\mathscr{H}^{1}(W)} \operatorname{card}\left(W \cap \partial_{L}^{u}(Z \cap L)\right)
$$

$\mathrm{E} \gamma^{+}(v)$ is independent of the choice of $W$. Obviously, $\gamma^{+}(v)$ requires only the knowledge of $u$ and $Z \cap L$. Moreover, $\gamma^{+}$is an unbiased estimator of the cosine transform of $\bar{S}(Z, \cdot)\left\llcorner u^{\oplus}\right.$, which can be shown using the concept of weighted surface processes in [19] or by more direct arguments given below. We present an alternative proof here.

Proposition 4.1. Let $Z$ be topologically regular $(Z=\mathrm{cl}$ int $Z)$, and assume that (2.6) holds. Using the above notation, we have

$$
\mathrm{E} \gamma^{+}(v)=\int_{u^{\oplus}}|\langle w, v\rangle| \bar{S}(Z, \mathrm{~d} w)=C\left[\bar{S}(Z, \cdot)\left\llcorner u^{\oplus}\right](v)\right.
$$

for all $v \in S^{d-1}$. 
Proof. Let $D^{1}$ and $D^{d-1}$ be relative open unit cubes in $g$ and $v^{\perp}$, respectively, and set $C^{d}:=\operatorname{cl}\left(D^{1}+D^{d-1}\right)$. We may assume that $W=D^{1}$. As $\partial_{L}^{u}(Z \cap L)=\left(\partial^{u} Z\right) \cap L$, we have

$$
\mathrm{E} \gamma^{+}(v)=\mathrm{E} \Theta^{\prime}\left(Z \cap g,\left(\partial^{u} Z \cap D^{1}\right) \times S^{1}(g)\right) .
$$

As support measures are defined locally, the stationarity of $Z$, Fubini's theorem, and (2.1) imply that

$$
\begin{aligned}
\mathrm{E} \gamma^{+}(v) & =\int_{v^{\perp}} \mathrm{E} \Theta^{\prime}\left([Z \cap g] \cap\left[C^{d}+z\right],\left(\partial^{u} Z \cap D^{1}\right) \times S^{1}(g)\right) \mathcal{H}^{d-1}(\mathrm{~d} z) \\
& =\mathrm{E} \int_{v^{\perp}} \Theta^{\prime}\left(\left[\left(Z \cap C^{d}\right)+z\right] \cap g,\left(\left[\partial^{u} Z+z\right] \cap D^{1}\right) \times S^{1}(g)\right) \mathcal{H}^{d-1}(\mathrm{~d} z) \\
& =\mathrm{E} \int_{\mathbb{R}^{d} \times S^{d-1}} \mathbf{1}_{x \in \partial^{u} Z} \mathbf{1}_{x \mid g \in D^{1}}\|w \mid g\| \Theta\left(Z \cap C^{d}, \mathrm{~d}(x, w)\right) \\
& =\mathrm{E} \int_{\left[\operatorname{int} C^{d} \cap \partial^{u} Z\right] \times S^{d-1}}|\langle w, v\rangle| \Theta\left(Z \cap C^{d}, \mathrm{~d}(x, w)\right) .
\end{aligned}
$$

In the last step we used the fact that $\Theta\left(Z \cap C^{d}, \cdot\right)$ vanishes outside $C^{d} \times S^{d-1}$ and the fact that $\Theta\left(Z \cap C^{d}, \cdot\right)$-almost all $(x, w)$ with $x \in D^{1} \times \partial D^{d-1}$ satisfy $w \in v^{\perp}$ and, therefore, do not contribute to the integral. Again using the fact that the support measures are defined locally, we may replace $Z \cap C^{d}$ by $Z$. As $Z=\operatorname{cl}$ int $Z, \mathscr{H}^{d-1}$-almost all $x \in \partial Z$ have a unique outer unit normal $w$ and $\mathbf{1}_{x \in \partial^{u} Z}=\mathbf{1}_{w \in u^{\oplus}}$ holds. Another application of Fubini's theorem and (2.8) yield

$$
\begin{aligned}
\mathrm{E} \gamma^{+}(v) & =\int_{u^{\oplus}}|\langle w, v\rangle| \mathrm{E}\left[\Theta\left(Z, \text { int } C^{d} \times \cdot\right)\right](\mathrm{d} w) \\
& =\int_{u^{\oplus}}|\langle w, v\rangle| \bar{S}(Z, \mathrm{~d} w) .
\end{aligned}
$$

Measurability of the involved functions in the preceding line of arguments follows from the weak continuity of support measures and the general considerations in [25, Anhang II].

We remark that Proposition 4.1 is still valid when $Z$ is not topologically regular, but the counts in the definition of $\gamma^{+}$must be modified: any isolated point of $g \cap \partial_{L}^{u}(Z \cap L)$ in $W$ must be counted twice, as it (almost surely) comes from a boundary point of $Z$ with exactly two antipodal outer normals. Proposition 4.1 implies that $\mathrm{E} \gamma^{+}$determines $\bar{S}(Z, \cdot)\left\llcorner u^{\oplus}\right.$ uniquely, as this measure is concentrated on a set without antipodal pairs. Replacing $u$ by $-u$, we can define a variable $\gamma^{-}(v)$ as in (4.1). It satisfies

$$
\mathrm{E} \gamma^{-}=C\left[\bar{S}(Z, \cdot)\left\llcorner u^{\ominus}\right] .\right.
$$

An estimation procedure for $\bar{S}(Z, \cdot)$ is now straightforward if we use known inversion algorithms from [6] and [12] to find a discrete approximation of a measure from finitely many (approximate) values of its cosine transform. Here we choose the least squares approach, as it is more robust against measurement errors than the linear program approach. To ease presentation, we restrict considerations to two-dimensional vertical sections in $\mathbb{R}^{3}$ and assume that $Z=\mathrm{cl}$ int $Z$. An extension to $k$-dimensional vertical sections for not necessarily topologically regular sets $Z \subset \mathbb{R}^{d}$ is straightforward, as long as $2 \leq k \leq d-1$.

(a) Choose a reference direction $u \in S^{2}$ such that $\bar{S}(Z, \cdot)\left\llcorner u^{\circ}=\boldsymbol{o}\right.$; see the comments after Corollary 3.1. 
(b) Choose $m \in \mathbb{N}$ vertical planes $L_{1}, \ldots, L_{m} \in \mathcal{L}_{2}^{3}(u)$, and consider independent realizations of the intersections $Z \cap L_{i}, i=1, \ldots, m$.

(c) In each $L_{i}$ choose $n \in \mathbb{N}$ test directions $v_{i 1}, \ldots, v_{i n} \in S^{1}\left(L_{i}\right)$ and determine the counts $\gamma^{-}\left(v_{i j}\right)$ and $\gamma^{+}\left(v_{i j}\right)$ of directed boundary points of $Z \cap L_{i}$ in unit intervals $j=1, \ldots, n$ and $i=1, \ldots, m$. Define the $m n$-dimensional vectors of observations

$$
\Gamma^{-}:=\left(\gamma^{-}\left(v_{i j}\right)\right)_{i j} \quad \text { and } \quad \Gamma^{+}:=\left(\gamma^{+}\left(v_{i j}\right)\right)_{i j} .
$$

According to (4.2) and (4.3), these vectors are unbiased estimators for

$$
\left([ C ( \overline { S } ( Z , \cdot ) \llcorner u ^ { \ominus } ) ] ( v _ { i j } ) ) _ { i j } \quad \text { and } \quad \left(\left[C\left(\bar{S}(Z, \cdot)\left\llcorner u^{\oplus}\right)\right]\left(v_{i j}\right)\right)_{i j},\right.\right.
$$

respectively.

(d) For $\mu \in \mathcal{M}_{+}$, set $\mu^{\ominus}:=\mu\left\llcorner u^{\ominus}\right.$ and

$$
C^{-}(\mu):=\left(C \mu^{\ominus}\left(v_{i j}\right)\right)_{i j} \in \mathbb{R}^{m n},
$$

and define $\boldsymbol{C}^{+}(\mu)$ analogously. Find solutions $\hat{\mu}_{m, n}^{-}$and $\hat{\mu}_{m, n}^{+}$of the optimization problems

$$
\text { minimize }\left\|\boldsymbol{C}^{-}(\mu)-\Gamma^{-}\right\| \text {subject to } \mu \in \mathcal{M}_{+} \text {being even }
$$

and

$$
\text { minimize }\left\|\boldsymbol{C}^{+}(\mu)-\Gamma^{+}\right\| \text {subject to } \mu \in \mathcal{M}_{+} \text {being even, }
$$

respectively. As these solutions are even measures whose cosine transforms best fit the measurements (in the least squares sense), they can be considered as estimators for the even parts of $\bar{S}(Z, \cdot)\left\llcorner u^{\ominus}\right.$ and $\bar{S}(Z, \cdot)\left\llcorner u^{\oplus}\right.$, respectively.

(e) The measure

$$
\hat{\mu}_{m, n}=2\left(\left(\hat{\mu}_{m, n}^{-}\left\llcorner u^{\ominus}\right)+\left(\hat{\mu}_{m, n}^{+}\left\llcorner u^{\oplus}\right)\right)\right.\right.
$$

is an estimator for $\bar{S}(Z, \cdot)$.

The infinite-dimensional least squares problems (4.4) and (4.5) can be discretized in a lossfree way; see, e.g. [6]. This means that among all the solutions of (4.4) (and similarly among those of (4.5)) there exists one with support in a finite set $T$ of prescribed directions, where $T$ depends only on the measurement directions $v_{i j}$. This allows us to replace (4.4) and (4.5) by quadratic programs with nonnegativity constraints. The latter can then be solved using standard software. Note that the resulting estimator $\hat{\mu}_{m, n}$ in (4.6) is not necessarily a mean normal measure of some stationary random set, as its centroid need not coincide with the origin. Asymptotically, however, $\hat{\mu}_{m, n}$ converges to the mean normal measure of $Z$ if the number of test directions $v_{i j}$ increases and these directions are chosen properly. To make this strong consistency result precise, we impose a condition on $v_{i j}$, which is slightly stronger than the denseness of the symmetrized sequence in $S^{2}$. Following [12, p. 14], the sequence

$$
\left(v_{i j}\right)_{i j}=\left(v_{11}, \ldots, v_{1 n}, v_{21}, \ldots, v_{2 n}, \ldots\right)
$$


is called asymptotically dense in $S^{2}$ if

$$
\liminf _{k \rightarrow \infty} \frac{1}{k} \operatorname{card}\left(F_{k} \cap G\right)>0
$$

for all origin-symmetric open sets $G \neq \varnothing$ in $S^{2}$. Here $F_{k}$ is the set of the first $k$ members of $\left(v_{i j}\right)$. Gardner et al. [6] discussed related notions.

Theorem 4.1. Let $Z$ be a stationary random set in $\&$ with $Z=\mathrm{cl}$ int $Z$, and let

$$
\mathrm{E}[N(Z \cap K)]^{2}<\infty
$$

for one (and hence all) convex body $K$ with interior points. Assume that $\left(v_{i j}\right)_{i j}$ is an asymptotically dense sequence in $S^{2}$ and that $n$ is fixed. Then, almost surely, the weak convergence

$$
\lim _{m \rightarrow \infty} \hat{\mu}_{m, n}=\bar{S}(Z, \cdot)
$$

holds.

Proof. Owing to (4.6), it is enough to show that $\mu_{m, n}^{+}$converges weakly and almost surely to the even part $v^{+}$of $\bar{S}(Z, \cdot)\left\llcorner u^{\oplus}\right.$ (and the corresponding result for $\mu_{m, n}^{-}$, which follows by replacing $u$ by $-u$ ). Note that (4.2) reads as $\mathrm{E} \gamma^{+}=C v^{+}$with this notation. The convergence claim is shown in several steps.

(i) The total masses $\mu_{m, n}^{+}\left(S^{2}\right)$ of $\mu_{m, n}^{+}$are almost surely uniformly bounded.

(ii) Let $\tau_{m}$ denote the sequence of even probability measures

$$
\tau_{m}:=\frac{1}{2 m n} \sum_{i=1}^{m} \sum_{j=1}^{n}\left(\delta_{v_{i j}}+\delta_{-v_{i j}}\right)
$$

and let $m^{\prime}$ be a subsequence of $m$ such that $\tau_{m^{\prime}}$ converges weakly to a measure $\tau$, say. Then, almost surely,

$$
\frac{1}{n} \sum_{i=1}^{n} \lim _{m \rightarrow \infty} \frac{1}{m^{\prime}} \sum_{i=1}^{m^{\prime}} f\left(v_{i j}\right) \gamma_{i j}^{+}=\int_{S^{2}}\left(C v^{+}\right) f \mathrm{~d} \tau
$$

for any even continuous function $f$ on the sphere.

(iii) If $\left(m^{\prime}\right)$ is a subsequence of $(m)$ such that a realization of $\mu_{m^{\prime}, n}^{+}$converges weakly to $\mu^{+}$ and $\tau_{m^{\prime}}$ to $\tau$, then

$$
\frac{1}{n} \sum_{i=1}^{n} \lim _{m \rightarrow \infty} \sum_{i=1}^{m^{\prime}}\left(C \mu_{m^{\prime}, n}^{+}\right)\left(v_{i j}\right) \gamma_{i j}^{+}=\int_{S^{2}}\left(C \mu^{+}\right)\left(C v^{+}\right) \mathrm{d} \tau .
$$

(iv) Excluding a zero measure set, we know that any accumulation point of $\mu_{m, n}^{+}$is $v^{+}$.

(v) Almost surely, $\mu_{m, n}^{+}$converges weakly to $v^{+}$. 
We start by showing step (ii) and writing $\|f\|_{\infty}$ for the maximum norm of $f$. Consider the independent random variables

$$
X_{j}:=\frac{1}{n} \sum_{i=1}^{n} f\left(v_{i j}\right) \gamma_{i j}^{+}
$$

Their variance can be bounded by

$$
\operatorname{var}\left(X_{j}\right) \leq \frac{\|f\|_{\infty}^{2}}{n^{2}} \mathrm{E}\left(\sum_{i=1}^{n} \gamma_{i j}^{+}\right)^{2} \leq\|f\|_{\infty}^{2} \max _{i=1}^{n} \mathrm{E}\left(\gamma_{i j}^{+}\right)^{2} .
$$

Here $\gamma_{i j}^{+}$counts (certain) boundary points of $Z$ in a unit line segment $s$, say. Assuming general position, a convex set can have at most two boundary points in $s$, so $\gamma_{i j}^{+} \leq 2 N(Z \cap s)$ and stationarity implies that

$$
\mathrm{E}\left(\gamma_{i j}^{+}\right)^{2} \leq 4 \mathrm{E}\left(N\left(Z \cap \frac{1}{2} B^{3}\right)\right)^{2} .
$$

By (4.7), the Kolmogorov criterion $\sum_{j=1}^{\infty} \operatorname{var}\left(X_{j}\right) / j^{2}<\infty$ is satisfied and $\left(X_{j}\right)$ obeys the strong law of large numbers. The zero measure set to be excluded in the strong law of large numbers can be chosen independent of $f$, as the Banach space of continuous functions on $S^{2}$ is separable.

Step (iii) follows from step (ii), as the weak convergence of $\mu_{m^{\prime \prime}, n}^{+}$to $\mu^{+}$implies uniform convergence of the corresponding cosine transforms; see, e.g. [23, Theorem 1.8.12].

To show step (i), we use the fact that $\mu_{m, n}^{+}$is a solution of (4.5). It therefore yields a better objective function value than the zero measure, so

$$
\left\|\boldsymbol{C}\left(\mu_{m, n}^{+}\right)-\Gamma^{+}\right\|^{2} \leq\left\|\Gamma^{+}\right\|^{2} .
$$

Let $c_{i j}=C\left(\mu_{m, n}^{+}\right)\left(v_{i j}\right)$ be the components of $\boldsymbol{C}^{+}\left(\mu_{m, n}^{+}\right)$. The Cauchy-Schwarz inequality yields

$$
\frac{1}{m n} \sum_{i j} c_{i j} \leq(m n)^{-1 / 2}\left\|\boldsymbol{C}^{+}\left(\mu_{m, n}^{+}\right)\right\| \leq\left(\frac{2}{m n} \sum_{i j} c_{i j} \gamma_{i j}^{+}\right)^{1 / 2} .
$$

As $v_{i j}$ is asymptotically dense, there exists a constant $c>0$ such that

$$
\frac{1}{c} \leq \frac{1}{m n} \sum_{i j}\left|\left\langle v_{i j}, \cdot\right\rangle\right|
$$

holds on $S^{2}$ for all sufficiently large $m$. The left-hand side of (4.8) is therefore bounded from below by $\mu_{m, n}^{+}\left(S^{2}\right) / c$. As $\left|\left\langle v_{i j}, \cdot\right\rangle\right| \leq 1$, the right-hand side of (4.8) can be estimated from above, and we obtain

$$
\mu_{m, n}^{+}\left(S^{2}\right) \leq \frac{2 c^{2}}{m n} \sum_{i j} \gamma_{i j}^{+}
$$

Again, the strong law of large numbers implies that the right-hand side of the last inequality is almost surely bounded.

To show step (iv), we fix a realization such that $\mu_{m, n}^{+}\left(S^{2}\right)$ is bounded. Owing to step (i), this holds for almost all realizations. Let $\mu^{+}$denote an arbitrary accumulation point of this 
sequence and assume that $\mu_{m^{\prime}, n}^{+}$converges to it. Extracting a subsequence $m^{\prime \prime}$ of $m^{\prime}$, we may assume that $\tau_{m^{\prime \prime}} \rightarrow \tau$.

By the definition of $\mu_{m^{\prime}, n}^{+}$, no even measure in $\mathcal{M}_{+}$can yield a better objective function value than $\mu_{m^{\prime}, n}^{+}$in (4.5). In particular, comparison with $v^{+}$yields

$$
\left\|\boldsymbol{C}\left(\mu_{m, n}^{+}\right)-\Gamma^{+}\right\|^{2} \leq\left\|\boldsymbol{C}\left(v^{+}\right)-\Gamma^{+}\right\|^{2} .
$$

This gives

$$
\frac{1}{m^{\prime \prime} n}\left(\left\|\boldsymbol{C}\left(\mu_{m^{\prime \prime}, n}^{+}\right)\right\|^{2}-\left\|\boldsymbol{C}\left(v^{+}\right)\right\|^{2}\right) \leq \frac{2}{m^{\prime \prime} n} \sum_{i j}\left(\left(C \mu_{m^{\prime \prime}, n}^{+}\right)\left(v_{i j}\right)-C v^{+}\left(v_{i j}\right)\right) \gamma_{i j}^{+} .
$$

In view of steps (ii) and (iii), letting $m$ tend to $\infty$ implies that

$$
\int_{S^{2}}\left(C \mu^{+}-C v^{+}\right)^{2} \mathrm{~d} \tau \leq 0
$$

As $\left(v_{i j}\right)$ is asymptotically dense in $S^{2}$, the support of $\tau$ is $S^{2}$, and thus $C \mu^{+}-C \nu^{+}=0$ on $S^{2}$. The two measures involved are even and, therefore, uniquely determined by their cosine transforms, which gives $\mu^{+}=v^{+}$, as required.

Finally, step (v) follows from the fact that a set of uniformly bounded measures is relatively compact in the weak topology. Hence, the convergence of $\mu_{m, n}^{+}$to $v^{+}$is equivalent to the fact that $v^{+}$is the only accumulation point of $\mu_{m, n}^{+}$. The latter was shown in step (iv).

The proof of Theorem 4.1 follows the approach introduced by Männle [16], who showed the consistency of a least squares estimator of a measure from cosine transforms under stochastic independence of the measurements. In general, independence of the sequence $\gamma_{i j}^{+}$can be assured only if each of the counts is obtained from an independent realization of $Z$-a sampling scheme, which is not realistic in practice. If long-range dependence in $Z$ is not present or negligible (as in the example of a Boolean model of convex grains with uniformly bounded diameter), this independence can (under ergodicity assumptions, approximately) be assured by placing the test line segments $W$ in the definition of $\gamma^{+}$far enough apart. We mention that the above convergence result can also be derived under stronger (but somewhat unrealistic) assumptions from [6, Section 9]. The speed of convergence result, also shown there, cannot be transferred directly to the present situation, as the restriction of measures in (4.6) is not a Lipschitz mapping in the Prohorov metric. Our last result shows that the requirements in Theorem 4.1 are in particular satisfied when $u, L_{1}, L_{2}, \ldots$, and the test directions are randomized. With this in mind, we denote the unique normalized Haar measure on $\mathcal{L}_{2}^{3}(u)$ by $v_{2}^{u}$.

Corollary 4.1. Let $Z$ be a stationary random set in $\&$ with $Z=\mathrm{cl}$ int $Z$ such that (4.7) holds. Assume that $n \in \mathbb{N}$ is fixed and that

(a) $u \in S^{2}$ is a random isotropic reference direction in $S^{2}$ independent of $Z$,

(b) given $u$, the planes $L_{1}, L_{2}, \ldots$ are independent and identically distributed (i.i.d.) in $\mathcal{L}_{2}^{3}(u)$ with distribution $v_{2}^{u}$ independent of $Z$,

(c) given $u$ and $L_{1}, L_{2}, \ldots$, for each $i=1,2, \ldots$, the directions $v_{i 1}, \ldots, v_{\text {in }}$ are i.i.d. isotropic random vectors in $S^{1}\left(L_{i}\right)$ independent of $Z$.

Then, almost surely, $\hat{\mu}_{m, n}$ converges weakly to $\bar{S}(Z, \cdot)$ as $m$ tends to $\infty$. 
Proof. In view of Theorem 4.1 it remains to show that the (random) sequence $v_{i j}$ is almost surely asymptotically dense in $S^{2}$. Given $u$, the distribution of $v_{11}$ is up to a normalizing constant the measure

$$
\int_{S^{2}} \mathbf{1}_{w \in \cdot}\left(1-\langle u, w\rangle^{2}\right)^{-1 / 2} \mathrm{~d} w,
$$

which has $S^{2}$ as its support. The strong law of large numbers now shows that $\left(v_{i j}\right)$ is almost surely asymptotically dense.

\section{Acknowledgement}

This research was supported by the Carlsberg Foundation and by the Danish Council for Strategic Research.

\section{References}

[1] Beneš, V. and Rataj, J. (2004). Stochastic Geometry: Selected Topics. Kluwer, Boston, MA.

[2] Beneš, V. AND SAXL, I. (2005). Stereological estimation of the rose of directions from the rose of intersections. In Recent Advances in Applied Probability, eds R. Baeza-Yates et al., Springer, New York, pp. 65-96.

[3] Fallert, H., Goodey, P. And Weil, W. (1997). Spherical projections and centrally symmetric sets. Adv. Math. 129, 301-322.

[4] Gardner, R. (2006). Geometric Tomography, 2nd edn. Cambridge University Press.

[5] Gardner, R. and Gritzmann, P. (1997). Discrete tomography: determination of finite sets by X-rays. Trans. Amer. Math. Soc. 349, 2271-2295.

[6] Gardner, R. J., Kiderlen, M. and Milanfar, P. (2006). Convergence of algorithms for reconstructing convex bodies and directional measures. Ann. Statist. 34, 1331-1374.

[7] Goodey, P., Kiderlen, M. And Weil, W. (1998). Section and projection means of convex bodies. Monatsh. Math. 126, 37-54.

[8] Goodey, P., Kiderlen, M. ANd Weil, W. (2008). Spherical projections and liftings in geometric tomography. Submitted.

[9] Gutkowski, P., Jensen, E. B. V. And Kiderlen, M. (2004). Directional analysis of digitized three-dimensional images by configuration counts. J. Microscopy 216, 175-185.

[10] Hug, D., LAst, G. AND WeIL, W. (2004). A local Steiner-type formula for general closed sets and applications. Math. Z. 246, 237-272.

[11] KIDERLEN, M. (1999). Schnittmittelungen und äquivariante Endomorphismen konvexer Körper. Doctoral Thesis, University of Karlsruhe.

[12] Kiderlen, M. (2001). Non-parametric estimation of the directional distribution of stationary line and fibre processes. Adv. Appl. Prob. 33, 6-24.

[13] Kiderlen, M. (2002). Determination of the mean normal measure from isotropic means of flat sections. $A d v$. Appl. Prob. 34, 505-519.

[14] Kiderlen, M. and Jensen, E. B. V. (2003). Estimation of the directional measure of planar random sets by digitization. Adv. Appl. Prob. 35, 583-602.

[15] Kiderlen, M. AND WeIl, W. (1999). Measure-valued valuations and mixed curvature measures of convex bodies. Geom. Dedicata 76, 291-329.

[16] MÄnnle, S. (2002). Ein Kleinste-Quadrat-Schätzer des Richtungsmaßes für stationäre Geradenprozesse. Master's Thesis. University of Karlsruhe.

[17] Molchanov, I. (1995). Statistics of the Boolean model: from the estimation of means to the estimation of distributions. Adv. Appl. Prob. 27, 63-86.

[18] Molchanov, I. and Stoyan, D. (1994). Directional analysis of fibre processes related to Boolean models. Metrika 41, 183-199.

[19] Pohlmann, S., Mecke, J. and Stoyan, D. (1981). Stereological formulas for stationary surface processes. Math. Operat. Statist. Ser. Statist. 12, 429-440.

[20] Rataj, J. (1996). Estimation of oriented direction distribution of a planar body. Adv. Appl. Prob. 28, $394-404$.

[21] RataJ, J. (1999). Translative and kinematic formulae for curvature measures of flat sections. Math. Nachr. 197, 89-101.

[22] Rataj, J. And Z̈̈HLe, M. (2001). Curvatures and currents for unions of sets with positive reach. II. Ann. Global Anal. Geom. 20, 1-21.

[23] Schneider, R. (1993). Convex Bodies: The Brunn-Minkowski Theory. Cambridge University Press. 
[24] Schneider, R. (2001). On the mean normal measures of a particle process. Adv. Appl. Prob. 33, $25-38$.

[25] SCHNEIDER, R. AND WeIL, W. (1992). Integralgeometrie. Teubner, Stuttgart.

[26] SchneIder, R. AND WeIL, W. (2000). Stochastische Geometrie. Teubner, Stuttgart.

[27] Stoyan, D. And Beneš, V. (1991). Anisotropy analysis for particle systems. J. Microsc. 164, 159-168.

[28] Stoyan, D., Kendall, W. and Mecke, J. (1995). Stochastic Geometry and its Applications, 2nd edn, John Wiley, New York.

[29] WeIL, W. (1982). Zonoide und verwandte Klassen konvexer Körper. Monatsh. Math. 94, 73-84.

[30] WeIL, W. (1995). The estimation of mean shape and mean particle number in overlapping particle systems in the plane. Adv. Appl. Prob. 27, 102-119.

[31] WeIL, W. (1997). Mean bodies associated with random closed sets. Suppl. Rend. Circ. Mat. Palermo Ser. II 50, 387-412.

[32] WeIL, W. (1997). The mean normal distribution of stationary random sets and particle processes. In Proc. Internat. Symp. Adv. Theory Appl. Random Sets (Fontainebleau, ed. D. Jeulin, 1986). World Scientific, Singapore, pp. 2133.

[33] WeIL, W. (1999). Intensity analysis of Boolean models. Pattern Recognition 32, 1675-1684.

[34] ZiEgEL, J. AND KiderLEN, M. (2008). Estimation of surface area and surface area measure of a three-dimensional set from digitizations. Submitted 